KYUNGPOOK Math. J. 53(2013), 397-406

http://dx.doi.org/10.5666/KMJ.2013.53.3.397

\title{
On SF-rings and Regular Rings
}

Tikaram Subedi* and Ardeline Mary Buhphang

Department of Mathematics, North Eastern Hill University, Shillong 793-022, In-

dia

e-mail : tsubedi2010@gmail.com and ardeline17@gmail.com

Abstract. A ring $R$ is called a left (right) SF-ring if simple left (right) $R$-modules are flat. It is still unknown whether a left (right) SF-ring is von Neumann regular. In this paper, we give some conditions for a left (right) SF-ring to be (a) von Neumann regular; (b) strongly regular; (c) division ring. It is proved that: (1) a right SF-ring $R$ is regular if maximal essential right (left) ideals of $R$ are weakly left (right) ideals of $R$ (this result gives an affirmative answer to the question raised by Zhang in 1994); (2) a left SF-ring $R$ is strongly regular if every non-zero left (right) ideal of $R$ contains a non-zero left (right) ideal of $R$ which is a W-ideal; (3) if $R$ is a left SF-ring such that $l(x)(r(x))$ is an essential left (right) ideal for every right (left) zero divisor $x$ of $R$, then $R$ is a division ring.

\section{Introduction}

Throughout this paper, $R$ denotes an associative ring with identity and all our modules are unitary. The symbols $J(R), Z\left({ }_{R} R\right)\left(Z\left(R_{R}\right)\right)$, $\operatorname{soc}\left({ }_{R} R\right)\left(\operatorname{soc}\left(R_{R}\right)\right)$ respectively stand for the Jacobson radical, left (right) singular ideal and left (right) socle of $R$. $R$ is semiprimitive if $J(R)=0$. $R$ is left non-singular if $Z\left({ }_{R} R\right)=0$. Right non-singular rings are defined similarly. For any $a \in R, l(a)(r(a))$ denotes the left (right) annihilator of $a$. By an ideal, we mean a two sided ideal. As usual, a reduced ring is a ring without non-zero nilpotent elements. $R$ is left (right) duo if every left (right) ideal of $R$ is an ideal. $R$ is a left quasi duo (MELT ) ring if every maximal (maximal essential) left ideal of $R$ is an ideal. Right quasi duo rings and MERT rings are defined similarly. $R$ is strongly left (right) bounded if every nonzero left (right) ideal of $R$ contains a non-zero ideal of $R([12])$. $R$ is a left (right) uniform ring if every non-zero left (right) ideal of $R$ is essential ([10]). $R$ is (von Neumann) regular if for every $a \in R$, there exists some $b \in R$ such that $a=a b a$. $R$ is strongly regular if for every $a \in R$, there exists some $b \in R$ such that $a=a^{2} b$. Clearly, $R$ is strongly regular if and only if $R$ is a reduced regular ring. Following [2], $R$ is left (right) weakly regular if for every left (right) ideal $I$ of $R, I=I^{2}$ and $R$

* Corresponding Author.

Received April 8, 2011; accepted July 24, 2012.

2010 Mathematics Subject Classification: 16D25, 16E50, 16D40.

Key words and phrases: Left SF-rings, von Neumann regular rings, strongly regular rings, weakly left ideals, W-ideals. 
is weakly regular if it is both left and right weakly regular. Clearly, a regular ring is weakly regular, but a weakly regular ring need not be regular (for example, see [2], Remark 6). Following [11], a left $R$-module $M$ is p-injective if for every principal left ideal $I$ of $R$ and every left $R$-homomorphism $f: I \longrightarrow M$, there exists some $m \in M$ such that $f(b)=b m$ for all $b \in I$ and $R$ is a left $P$ - $V$-ring if every simple left $R$-module is p-injective. Again, following [11], a left (right) $R$-module $M$ is $Y J$-injective if for each $0 \neq a \in R$, there exists a positive integer $n$ such that $a^{n} \neq 0$ and every left (right) $R$-homomorphism from $R a^{n}\left(a^{n} R\right)$ to $M$ extends to a left (right) $R$-homomorphism from $R$ to $M$ and $R$ is a left (right) $G P$ - $V$-ring if simple left (right) $R$-modules are YJ-injective. A regular ring is left (right) GP-V-ring (see, [9], Lemma 2) but a left (right) GP-V-ring need not be regular (see, [7]). Following [3], $R$ is a left (right) SF-ring if simple left (right) $R$-modules are flat. It is well known that regular rings are left (right) SF-rings. As far as we know, the question that whether left (right) SF-rings are necessarily regular, is still open. Over the last three and a half decades, left (right) SF-rings have been studied by many authors and the regularity of left (right) SF-rings which satisfy certain additional conditions is proved (cf. for example, [3], [4], [8], [10]-[15]).

We recall the following two definitions following [11]:

Definition 1.1. An additive subgroup $L$ of a ring $R$ is a weakly left ideal of $R$ if for every $x \in L$ and every $r \in R$ there exists a positive integer $n$ such that $(r x)^{n} \in L$. The notion of a weakly right ideal of a ring is defined similarly.

Definition 1.2. A ring $R$ is an $L W-$ ring ( $R W-$ ring) if every left (right) ideal of $R$ is a weakly right (left) ideal of $R$.

Example 1.3. Let $R=U T_{2}(\mathbb{Q})$, the ring of upper triangular matrices over $\mathbb{Q}$. Take $L=\left\{\left(\begin{array}{ll}a & 0 \\ 0 & 0\end{array}\right): a \in \mathbb{Q}\right\}$ and $K=\left\{\left(\begin{array}{ll}0 & 0 \\ 0 & a\end{array}\right): a \in \mathbb{Q}\right\}$. Then $L$ is a left ideal and $K$ is a right ideal of $R$. It is easy to see that $L$ is not a weakly right ideal and $K$ is not a weakly left ideal of $R$.

Example 1.4. Take $R=\left\{\left(\begin{array}{cccc}a & a_{1} & a_{2} & a_{3} \\ 0 & a & a_{4} & a_{5} \\ 0 & 0 & a & a_{6} \\ 0 & 0 & 0 & a\end{array}\right): a, a_{i} \in \mathbb{Z}_{2}, i=1,2,3,4,5,6\right\}$.

Let $r \in R$. Suppose $A$ is any left ideal of $R$. If $A=R$, then $A$ is a weakly right ideal of $R$. If $A \neq R$ and $x \in A$, then $x=\left(\begin{array}{cccc}0 & x_{1} & x_{2} & x_{3} \\ 0 & 0 & x_{4} & x_{5} \\ 0 & 0 & 0 & x_{6} \\ 0 & 0 & 0 & 0\end{array}\right)$ for some

$x_{i} \in \mathbb{Z}_{2}, i=1,2,3,4,5,6$. Therefore $(x r)^{4}=0$. This implies that $A$ is a weakly right ideal of $R$. It follows that $R$ is an LW-ring.

Suppose $B \neq R$ be any right ideal of $R$. It is easy to see that for every 
$y \in B,(r y)^{4}=0$. Hence $B$ is a weakly left ideal of $R$. Therefore $R$ is an RWring.

Let $L=\left\{\left(\begin{array}{cccc}0 & b_{1} & b_{1} & b_{2} \\ 0 & 0 & 0 & b_{3} \\ 0 & 0 & 0 & b_{4} \\ 0 & 0 & 0 & 0\end{array}\right): b_{i} \in \mathbb{Z}_{2}, i=1,2,3,4\right\}$.

Then $L$ is a left ideal of $R$. Now

$$
\left(\begin{array}{llll}
0 & 1 & 1 & 0 \\
0 & 0 & 0 & 0 \\
0 & 0 & 0 & 0 \\
0 & 0 & 0 & 0
\end{array}\right) \in L,\left(\begin{array}{cccc}
0 & 0 & 0 & 0 \\
0 & 0 & 1 & 0 \\
0 & 0 & 0 & 0 \\
0 & 0 & 0 & 0
\end{array}\right) \in R
$$

and

$$
\left(\begin{array}{llll}
0 & 1 & 1 & 0 \\
0 & 0 & 0 & 0 \\
0 & 0 & 0 & 0 \\
0 & 0 & 0 & 0
\end{array}\right)\left(\begin{array}{llll}
0 & 0 & 0 & 0 \\
0 & 0 & 1 & 0 \\
0 & 0 & 0 & 0 \\
0 & 0 & 0 & 0
\end{array}\right)=\left(\begin{array}{llll}
0 & 0 & 1 & 0 \\
0 & 0 & 0 & 0 \\
0 & 0 & 0 & 0 \\
0 & 0 & 0 & 0
\end{array}\right) \notin L
$$

It follows that $L$ is not an ideal of $R$ and hence $R$ is not left duo.

Also, $K=\left\{\left(\begin{array}{cccc}0 & 0 & 0 & 0 \\ 0 & 0 & b_{1} & b_{2} \\ 0 & 0 & 0 & 0 \\ 0 & 0 & 0 & 0\end{array}\right): b_{i} \in \mathbb{Z}_{2}, i=1,2\right\}$ is a right ideal of $R$.

However

$$
\left(\begin{array}{llll}
0 & 1 & 0 & 0 \\
0 & 0 & 0 & 0 \\
0 & 0 & 0 & 0 \\
0 & 0 & 0 & 0
\end{array}\right)\left(\begin{array}{llll}
0 & 0 & 0 & 0 \\
0 & 0 & 1 & 0 \\
0 & 0 & 0 & 0 \\
0 & 0 & 0 & 0
\end{array}\right)=\left(\begin{array}{llll}
0 & 0 & 1 & 0 \\
0 & 0 & 0 & 0 \\
0 & 0 & 0 & 0 \\
0 & 0 & 0 & 0
\end{array}\right) \notin K .
$$

We see that $K$ is not an ideal of $R$. Therefore $R$ is not a right duo ring.

\section{SF-rings and Weakly One Sided Ideals}

In this section, as a continuation of [11], we give further characterizations of strongly regular rings via weakly one sided ideals. We also prove the regularity of right SF-rings whose maximal essential right (left) ideals are weakly left (right) ideals.

We start with the following observation:

Proposition 2.1. The following conditions are equivalent for a ring $R$ :

(1) $R$ is an LW-ring.

(2) Every principal left ideal of $R$ is a weakly right ideal. 
(3) Every finitely generated left ideal of $R$ is a weakly right ideal.

Proof. It is clear that $(1) \Longrightarrow(2)$ and $(1) \Longrightarrow(3)$.

$(2) \Longrightarrow(1)$. Let $L$ be a left ideal of $R$ and $a \in L, r \in R$. By hypothesis, $R a$ is a weakly right ideal. Hence there exists a positive integer $n$ such that $(a r)^{n} \in R a \subseteq L$. This proves that $L$ is a weakly right ideal of $R$ so that $R$ is an LW-ring.

$(3) \Longrightarrow(1)$ can be proved similarly.

Lemma 2.2. Let $R$ be a semiprimitive ring whose maximal left ideals are weakly right ideals, then $R$ is reduced.

Proof. Suppose $0 \neq a \in R$ such that $a^{2}=0$, then $a \notin J(R)$. So $a \notin M$ for some maximal left ideal $M$ of $R$. Hence $M+R a=R$ implying $M a=R a$ which yields $a=b a$ for some $b \in M$. We therefore get $x+r b a=1$ for some $x \in M, r \in R$. As $M$ is a weakly right ideal and $r b \in M$, there exists a positive integer $k$ such that $(r b a)^{k} \in M$ and so $(1-x)^{k} \in M$. Using $x \in M$ in $(1-x)^{k} \in M$ we get $1 \in M$ which contradicts that $M \neq R$. Therefore $R$ is reduced.

Similarly, we can prove Lemma 2.3 .

Lemma 2.3. Let $R$ be a semiprimitive ring whose maximal right ideals are weakly left ideals, then $R$ is reduced.

Lemma 2.4([4], Proposition 3.2). Let $R$ be a left (right) SF-ring and I be an ideal of $R$. Then $R / I$ is also a left (right) SF-ring.

Lemma 2.5([4], Remark 3.13). Let $R$ be a reduced left (right) SF-ring. Then $R$ is strongly regular.

Lemma 2.6([4], Theorem 4.10). A left (right) quasi duo left SF-ring is strongly regular.

Lemma 2.7([4], Proposition 4.3). Let $R$ be a reduced ring. Then $R$ is left weakly regular if and only if $R$ is right weakly regular.

Lemma 2.8([5], Lemma 2.1). If $R$ is a left (right) GP-V-ring, then $J(R)=0$.

The following lemma can be proved easily.

Lemma 2.9. Let $R$ be a ring and $I$ an ideal of $R$. For every left (right) ideal $K$ of $R$ such that $I \subseteq K, K$ is a weakly right (left) ideal of $R$ if and only if $K / I$ is a weakly right (left) ideal of $R / I$.

Zhang in [11] proposed the following question: Is $R$ von Neumann regular if it is a left SF-ring whose every maximal right (left) ideal is a weakly left (right) ideal of $R$ ? Theorem 2.10 not only gives an affirmative answer to this question but also gives generalizations of many other results established in [11].

Theorem 2.10. Let $R$ be a ring whose maximal left (right) ideals are weakly right (left) ideals, then the following conditions are equivalent: 
(1) $R$ is a left GP-V-ring.

(2) $R$ is a left weakly regular ring.

(3) $R$ is a left $S F$-ring.

(4) $R$ is a right $G P$-V-ring.

(5) $R$ is a right weakly regular ring.

(6) $R$ is a right $S F$-ring.

(7) $R$ is a regular ring.

(8) $R$ is a strongly regular ring.

Proof. Let $R$ be a ring whose maximal left ideals are weakly right ideals. That $(8) \Longrightarrow(1),(2),(3),(4),(5),(6),(7)$ are well known.

$(1) \Longrightarrow(8)$. $R$ is reduced by Lemma 2.8 and Lemma 2.2. Let $a \in R$. If $l(a)+R a \neq$ $R$, then it must be contained in a maximal left ideal $M$ of $R$. Since $R$ is a left GP-V-ring, $R / M$ being a simple left $R$-module is YJ-injective. Hence there exists a positive integer $n$ such that $a^{n} \neq 0$ and every left $R$-homomorphism from $R a^{n}$ to $R / M$ extends to one from $R$ to $R / M$. Define $f: R a^{n} \longrightarrow R / M$ by $f\left(r a^{n}\right)=r+M$ for every $r \in R$. As $R$ is reduced, $l\left(a^{n}\right)=l(a)$ so that $f$ is well-defined. It follows that $1+M=a^{n}(b+M)$ for some $b \in R$ which yields $1-a^{n} b \in M$. If $a^{n} b \notin M$, then $M+R a^{n} b=R$. This gives $x+r a^{n} b=1$ for some $x \in M, r \in R$. As $M$ is a weakly right ideal and $r a^{n} \in M$, there exists some $k>0$ such that $\left(r a^{n} b\right)^{k} \in M$, that is $(1-x)^{k} \in M$, whence $1 \in M$, a contradiction to $M \neq R$. Therefore $l(a)+R a=R$. This implies that there exists some $x \in R, y \in l(a)$ such that $x a+y=1$ which yields $a=x a^{2}$. Hence $R$ is strongly regular.

$(2) \Longrightarrow(8)$. Let $a \in R$. If $l(a)+R a \neq R$, then there exists a maximal left ideal $M$ of $R$ containing $l(a)+R a$. As $R$ is left weakly regular, $R a=R a R a$ which gives $a=\sum r_{i} a s_{i} a$ for some $r_{i} \in R, s_{i} \in R$. So $1-\sum r_{i} a s_{i} \in l(a) \subseteq M$. Suppose $\sum r_{i} a s_{i} \notin M$, then $r_{k} a s_{k} \notin M$ for some $k$. Therefore $M+R r_{k} a s_{k}=R$ and hence $x+r r_{k} a s_{k}=1$ for some $x \in M, r \in R$. As $M$ is a weakly right ideal and $r r_{k} a \in M$, there exists a positive integer $n$ such that $\left(r r_{k} a s_{k}\right)^{n} \in M$ so that $(1-x)^{n} \in M$, whence $1 \in M$. This contradicts that $M \neq R$. Thus $l(a)+R a=R$. This implies that $R$ is strongly regular.

$(3) \Longrightarrow(8)$. Let $\bar{R}=R / J(R)$. Then $\bar{R}$ is a semiprimitive ring. Also, by hypothesis and Lemma 2.9, every maximal left ideal of $\bar{R}$ is a weakly right ideal of $\bar{R}$. Therefore it follows from Lemma 2.2, Lemma 2.4 and Lemma 2.5 that $\bar{R}$ is strongly regular. Therefore $\bar{R}$ is left duo so that $R$ is left quasi duo. Hence by Lemma $2.6, R$ is strongly regular.

$(6) \Longrightarrow(8)$ can be proved similarly.

$(4) \Longrightarrow(8)$. Let $a \in R$. $R$ is reduced by Lemma 2.8 and Lemma 2.2 and therefore $r(a)=l(a)$. If $r(a)+R a \neq R$, then it must be contained in some maximal left ideal $L$ of $R$. We claim that $R a R \subseteq L$. If this is not true, then ras $\notin L$ for some $r \in R, s \in R$. Then $L+\operatorname{Rras}=R$ which yields $b+\operatorname{tras}=1$ for some $b \in L, t \in R$. Since $L$ is a weakly right ideal and $\operatorname{tra} \in L$, there exists some positive integer $n$ such 
that $(\text { tras })^{n} \in L$, that is $(1-b)^{n} \in L$, whence $1 \in L$ which contradicts that $L \neq R$. Therefore $R a R \subseteq L$ and so $r(a)+R a R \subseteq L \neq R$. Hence there exists a maximal right ideal $M$ of $R$ such that $r(a)+R a R \subseteq M$. Since $R$ is a right GP-V-ring, $R / M$ is YJ-injective. Thus there exists a positive integer $m$ such that $a^{m} \neq 0$ and every right $R$-homomorphism from $a^{m} R$ to $R / M$ extends to one from $R$ to $R / M$. Define $f: a^{m} R \longrightarrow R / M$ by $f\left(a^{m} r\right)=r+M$ for every $r \in R$. As $R$ is reduced, $r\left(a^{m}\right)=r(a)$ so that $f$ is a well-defined. Therefore we get $1+M=(b+M) a^{m}$ for some $b \in R$ which yields $1-b a^{m} \in M$. But $b a^{m} \in R a R \subseteq M$, whence $1 \in M$. This contradiction shows that $r(a)+R a=R$. Therefore $x+y a=1$ for some $x \in r(a)$ and $y \in R$ which yields $a=$ aya. Thus $R$ is regular. As $R$ is reduced also, $R$ is strongly regular.

$(7) \Longrightarrow(2)$ is well known.

$(5) \Longrightarrow(2)$. Since $R$ is right weakly regular, it is semiprimitive. Thus by Lemma 2.2 and Lemma $2.7, R$ is left weakly regular.

We can similarly prove the theorem for a ring $R$ whose maximal right ideals are weakly left ideals.

Corolary 2.11([11], Theorem 2). A ring $R$ is strongly regular if and only if $R$ is a left $P$ - $V$-ring and every maximal left ideal of $R$ is a weakly right ideal of $R$.

Corolary 2.12([11], Theorem 3). The following conditions are equivalent:

(1) $R$ is a strongly regular ring.

(2) $R$ is an LW left SF-ring.

(3) $R$ is an $L W$ right $S F$-ring.

Corolary 2.13([11], Theorem 6). The following conditions are equivalent:

(1) $R$ is a strongly regular ring.

(2) $R$ is an $L W$ left $G P-V$-ring.

(3) $R$ is an $R W$ right $G P$ - $V$-ring.

Lemma 2.14([12], Theorem 1). An MERT right SF-ring is regular.

Lemma 2.15([8], Proposition 2.2). An MERT left SF-ring is regular.

Theorem 2.16. If $R$ is a right $S F$-ring whose maximal essential right ideals are weakly left ideals, then $R$ is regular.

Proof. Let $\bar{R}=R / \operatorname{soc}\left(R_{R}\right)$. Then by Lemma $2.4, \bar{R}$ is a right SF-ring. Let $T$ be a maximal right ideal of $\bar{R}$. Then $T=M / \operatorname{soc}\left(R_{R}\right)$ for some maximal right ideal $M$ of $R$ such that $\operatorname{soc}\left(R_{R}\right) \subseteq M$. It is clear that $M$ is an essential right ideal of $R$. By hypothesis, $M$ is a weakly left ideal of $R$. Thus by Lemma 2.9, $T$ is a weakly left ideal of $\bar{R}$. This implies that $\bar{R}$ is a ring whose maximal right ideals are weakly left ideals. Therefore by Theorem $2.10, \bar{R}$ is strongly regular so that $R$ is an MERT ring. Hence by Lemma $2.14, R$ is regular. 
Similarly, considering $\bar{R}=R / \operatorname{soc}\left({ }_{R} R\right)$ and using Lemma 2.4, Lemma 2.9, Theorem 2.10 and the dual of Lemma 2.15, we can prove the following theorem:

Theorem 2.17. If $R$ is a right SF-ring whose maximal essential left ideals are weakly right ideals, then $R$ is regular.

\section{SF-rings and W-ideals}

Following [15], a left ideal $L$ of a ring $R$ is called a weak-ideal $(W-i d e a l)$ if for every $0 \neq a \in L$, there exists some $n>0$ such that $a^{n} \neq 0$ and $a^{n} R \subseteq L$. A right ideal $K$ of $R$ is defined similarly to be a $W-i d e a l$. By ([15], Example 1.2), the ring $R$ of Example 1.4 is a ring in which \{ideals of $R\} \subsetneq\{\mathrm{W}$-ideals of $R\}$.

In this section, we study the strong regularity of left (right) SF-rings via Wideals.

For ease of reference, we first quote the following two lemmas.

Lemma 3.1([4], Lemma 3.14). Let $L$ be a left ideal of $R$. Then $R / L$ is a flat left $R$-module if and only if for every $a \in L$, there exists some $b \in L$ such that $a=a b$.

Lemma 3.2([13], Lemma 9). Let $R$ be a left or a right SF-ring. If $R / Z\left({ }_{R} R\right)$ is a reduced ring, then $R$ is strongly regular.

We now state and prove the main result of this section as follows:

Theorem 3.3. The following conditions are equivalent for a ring $R$ :

(1) $R$ is strongly regular.

(2) $R$ is a left $S F$-ring such that every non-zero left ideal of $R$ contains a non-zero left ideal of $R$ which is a $W$-ideal.

(3) $R$ is a left $S F$-ring such that every non-zero right ideal of $R$ contains a non-zero right ideal of $R$ which is a $W$-ideal.

(4) $R$ is a right SF-ring such that every non-zero left ideal of $R$ contains a non-zero left ideal of $R$ which is a $W$-ideal.

(5) $R$ is a right $S F$-ring such that every non-zero right ideal of $R$ contains a nonzero right ideal of $R$ which is a $W$-ideal.

Proof. It is well known that (1) $\Longrightarrow(2),(3),(4)$ and (5).

$(2) \Longrightarrow(1)$. Suppose $0 \neq a \in Z\left({ }_{R} R\right)$, then $l(a)$ is an essential left ideal of $R$. By hypothesis, there exists a non-zero left ideal $I$ of $R$ which is a W-ideal and $I \subseteq R a$. Let $0 \neq b \in I$, then there exists some $n>0$ such that $b^{n} \neq 0$ and $b^{n} R \subseteq I \subseteq R a$. We claim that $R a+r\left(b^{n} R\right)=R$. If this is not true, then there exists a maximal left ideal $M$ of $R$ such that $R a+r\left(b^{n} R\right) \subseteq M$. Then by Lemma 3.1, there exists some $c \in M$ such that $a=a c$, that is $1-c \in r(a) \subseteq r\left(b^{n} R\right) \subseteq M$, whence $1 \in M$, a contradiction. Therefore $R a+r\left(b^{n} R\right)=R$ which implies $x a+y=1$ for some $x \in R, y \in r\left(b^{n} R\right)$ yielding $b^{n} x a=b^{n}$. As $x a \in Z\left({ }_{R} R\right), l(x a) \cap R b^{n} \neq 0$. Let $0 \neq z b^{n} \in l(x a) \cap R b^{n}$. Then $z b^{n}=z b^{n} x a=0$, a contradiction to $z b^{n} \neq 0$. 
Therefore $Z\left({ }_{R} R\right)=0$.

We now prove that $R$ is reduced. Suppose $0 \neq a \in R$ such that $a^{2}=0$. Then $a \notin Z\left({ }_{R} R\right)$. So there exists a non-zero left ideal $L$ of $R$ such that $l(a) \oplus L$ is an essential left ideal of $R$. By hypothesis, there exists a non-zero left ideal $T$ of $R$ which is a $\mathrm{W}$-ideal and $T \subseteq L$. Let $0 \neq b \in T$. There exists a positive integer $n$ such that $b^{n} \neq 0$ and $b^{n} R \subseteq T$. Then

$$
b^{n} R a \subseteq b^{n} R \cap R a \subseteq T \cap l(a) \subseteq L \cap l(a)=0 .
$$

This implies $b^{n} R \subseteq L \cap l(a)=0$ implying $b^{n}=0$. This contradiction shows that $R$ is reduced and hence by Lemma $2.5, R$ is strongly regular.

Similarly $(5) \Longrightarrow(1)$.

$(3) \Longrightarrow(1)$. Suppose $a \notin Z\left(R_{R}\right)$ such that $a^{2} \in Z\left(R_{R}\right)$, then there exists a nonzero right ideal $K$ of $R$ such that $r(a) \oplus K \subseteq r\left(a^{2}\right)$. By hypothesis, there exists a non-zero right ideal $I$ of $R$ such that $I \subseteq K$ and $I$ is a W-ideal. Let $0 \neq b \in I$. There exists some $n>0$ such that $b^{n} \neq 0$ and $R b^{n} \subseteq I \subseteq K$. Hence

$$
a R b^{n} \subseteq r(a) \cap R b^{n} \subseteq r(a) \cap K=0 .
$$

Therefore $R b^{n} \subseteq r(a) \cap K=0$ which implies that $b^{n}=0$, a contradiction to $b^{n} \neq 0$. Thus $R / Z\left(R_{R}\right)$ is reduced and therefore by dual of Lemma 3.2, $R$ is strongly regular. Similarly $(4) \Longrightarrow(1)$.

Corollary 3.4([12], Theorem 3). The following conditions are equivalent:

(1) $R$ is a strongly regular ring.

(2) $R$ is a strongly left bounded left SF-ring.

(3) $R$ is a strongly right bounded left SF-ring.

(4) $R$ is a strongly left bounded right SF-ring.

(5) $R$ is a strongly right bounded right SF-ring.

\section{SF-rings and Division Rings}

In this section, we give some conditions for a left SF-ring to be a division ring. Our first main result of this section is the following:

Theorem 4.1. Let $R$ be a left SF-ring such that $l(x)$ is an essential left ideal of $R$ for every right zero divisor $x$ of $R$, then $R$ is a division ring.

Proof. Suppose $a^{2} \in Z\left({ }_{R} R\right)$ such that $a \notin Z\left({ }_{R} R\right)$. If $\operatorname{Rr}(a)+Z\left({ }_{R} R\right)=R$, then $a=$ $b a+\sum r_{i} t_{i} a$, where $b \in Z\left({ }_{R} R\right), r_{i} \in R, t_{i} \in r(a), t_{i} a \neq 0$ for each $i$. Now, for each $i,\left(t_{i} a\right)^{2}=t_{i}\left(a t_{i}\right) a=0$ which implies $t_{i} a \in l\left(t_{i} a\right)$ so that $l\left(t_{i} a\right) \neq 0$. By hypothesis, $l\left(t_{i} a\right)$ is an essential left ideal of $R$, that is $t_{i} a \in Z\left({ }_{R} R\right)$. Therefore it follows that $a \in Z\left({ }_{R} R\right)$ which contradicts that $a \notin Z\left({ }_{R} R\right)$. Hence $\operatorname{Rr}(a)+Z\left({ }_{R} R\right) \neq R$ and so there exists a maximal left ideal $M$ of $R$ such that $\operatorname{Rr}(a)+Z\left({ }_{R} R\right) \subseteq M$. Since $R$ is a left SF-ring and $a^{2} \in Z\left({ }_{R} R\right) \subseteq M$, by Lemma 3.1, there exists some $c \in M$ 
such that $a^{2}=a^{2} c$, that is $a-a c \in r(a) \subseteq M$, whence $a \in M$. Hence again by Lemma 3.1, there exists some $d \in M$ such that $a=a d$. Then $1-d \in r(a) \subseteq M$ so that $1 \in M$, contradicting $M \neq R$. Therefore $R / Z\left({ }_{R} R\right)$ is reduced and hence by Lemma $3.2, R$ is strongly regular. Since a strongly regular ring is left non-singular, it follows that $Z\left({ }_{R} R\right)=0$ and therefore by hypothesis, $l(w)=0$ for all $0 \neq w \in R$. Let $0 \neq u \in R$. If $R u \neq R$, let $L$ be a maximal left ideal of $R$ such that $R u \subseteq L$. As $R$ is a left SF-ring, by Lemma 3.1, there exists some $v \in L$ such that $u=u v$, that is $u \in l((1-v))$. Since $u \neq 0$, it follows that $1-v=0$, that is $v=1$. Therefore $1 \in L$ which is a contradiction to $L \neq R$. Thus $R u=R$. This proves that $R$ is a division ring.

Corollary 4.2. The following conditions are equivalent for a ring $R$ :

(1) $R$ is a division ring.

(2) $R$ is a left uniform, left SF-ring.

Lemma 4.3([8], Lemma $1.2(2))$. If $R$ is a left SF-ring, then $Z\left(R_{R}\right) \subseteq J(R)$.

We now give another main result of this section.

Theorem 4.4. Let $R$ be a left $S F$-ring such that $r(x)$ is an essential right ideal of $R$ for every left zero divisor $x$ of $R$, then $R$ is a division ring.

Proof. Let $a^{2} \in J(R)$ such that $a \notin J(R)$. If $\operatorname{Rr}(a)+J(R)=R$, then $a=b a+\sum r_{i} t_{i} a$ where $b \in J(R), r_{i} \in R, t_{i} \in r(a)$ and $t_{i} a \neq 0$ for each $i$. Now for each $i,\left(t_{i} a\right)^{2}=t_{i}\left(a t_{i}\right) a=0$ which implies $t_{i} a \in r\left(t_{i} a\right)$ so that $r\left(t_{i} a\right) \neq 0$. By hypothesis, $t_{i} a \in Z\left(R_{R}\right)$. Then by Lemma $4.3, t_{i} a \in J(R)$. Therefore it follows that $a \in J(R)$ which is a contradiction. Hence $\operatorname{Rr}(a)+J(R) \neq R$ so that there exists a maximal left ideal $M$ of $R$ such that $\operatorname{Rr}(a)+J(R) \subseteq M$. Since $a^{2} \in J(R) \subseteq M$, by Lemma 3.1, there exists some $c \in M$ such that $a^{2}=a^{2} c$. Following the proof of Theorem 4.1, we get a contradiction. This proves that $R / J(R)$ is reduced. Hence by Lemma 2.4 and Lemma $2.5, R / J(R)$ is strongly regular so that $R$ is left quasi duo. Therefore by Lemma $2.6, R$ is strongly regular. This yields $Z\left(R_{R}\right)=0$, since a strongly regular ring is right non-singular. Then by hypothesis, it follows that $r(w)=0$ for all $0 \neq w \in R$. Let $0 \neq u \in R$. If $R u \neq R$, there exists a maximal left ideal $L$ of $R$ such that $R u \subseteq L$. Since $R$ is left SF-ring, there exists some $v \in R$ such that $u(1-v)=0$. Then it follows that $u=0$ or $v=1$ which is a contradiction. Therefore $R u=R$ and $R$ is a division ring.

Corollary 4.5([10], Theorem 6). The following conditions are equivalent for a ring $R$ :

(1) $R$ is a division ring.

(2) $R$ is a left uniform, right SF-ring.

Acknowledgements. The authors thank the referee for his/her helpful comments. 


\section{References}

[1] F. W. Anderson and K. R. Fuller, Rings and Categories of Modules (Graduate Text in Mathematics 13), Springer -Verlag, Berlin Heldelberg-New York, (1974).

[2] V. S. Ramamurthy, Weakly regular rings, Canad. Math. Bull., 16(3)(1973), 317-321.

[3] V. S. Ramamurthy, On the injectivity and flatness of certain cyclic modules, Proc. Amer. Math. Soc., 48(1975), 21-25.

[4] M. B. Rege, On von Neumann regular rings and SF-rings, Math. Japonica, 31(6)(1986), 927-936.

[5] X. Song and X. Yin, Generalizations of V-rings, Kyungpook Math. J., 45(2005), 357-362.

[6] B. T. Strenstrom, Rings of quotients- An introduction to the methods of ring theory, Springer-Verlag, New York, (1975).

[7] G. Xiao, On GP-V-rings and characterizations of strongly regular rings, Northeast. Math. J., 18(4)(2002), 291-297.

[8] Y. Xiao, One sided SF-rings with certain chain conditions, Canad. Math. Bull., 37(2)(1994), 272-277.

[9] R. Yue. Chi. Ming, On von Neumann regular rings, Proc. Edinburgh Math. Soc., 19(1974), 89-91.

[10] R. Yue. Chi. Ming, On von Neumann regular rings, VIII, J. Korean Math. Soc., 19 (2) (1983), 97-104.

[11] J. Zhang, Characterizations of strongly regular rings, Northeast. Math. J., 10(3)(1994), 359-364.

[12] J. Zhang and X. Du, Von Neumann regularity of SF-rings, Communications in Algebra, 21(7)(1993), 2445-2451.

[13] J. Zhang and R. Lu, Strongly regular rings and SF-rings, Northeast Math. J., 14(1)(1998), 61-68.

[14] H. Zhou and X. Wang, Von Neumann regular rings and right SF-rings, Northeast Math. J., 20(2)(2004a), 75-78.

[15] H. Zhou, Left SF-rings and regular rings, Communications in Algebra, 35(2007), 3842-3850. 